\title{
Aging alteration of skin T cells is different from that of blood T cells
}

Hanako Koguchi-Yoshioka ${ }^{1,2}$, Elena Hoffer ${ }^{3}$, Stanley Cheuk ${ }^{3}$, Yutaka Matsumura' ${ }^{2}, \mathrm{Sa} \mathrm{Vo}^{2}$, Yasuhiro Fujisawaㄹ, Manabu Fujimoto, ${ }^{1,2}$, Liv Eidsmo ${ }^{3}$, Rachael A. Clark', Rei Watanabe2

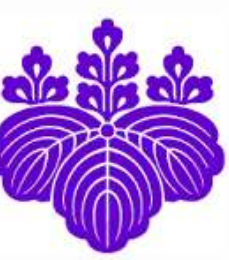

${ }^{1}$ Department of Dermatology, Graduate School of Medicine, Osaka University, Japan.

2 Department of Dermatology, Faculty of Medicine, University of Tsukuba, Japan.

${ }^{3}$ Department of Medicine Solna, Karolinska Institutet and Karolinska University Hospital, Sweden.

${ }^{4}$ Department of Dermatology, Brigham and Women's Hospital, Boston, USA.

\section{Background}

Aging of the immune system has been appreciated mainly from the aspect of circulating cells ${ }^{1}$. However, the aging alteration of skin $\mathrm{T}$ cells has been poorly revealed even though skin is an inevitable barrier tissue and resent researches have demonstrated the barrier tissue-specific development of resident memory $T$ cells $\left(T_{R M}\right)^{2-4}$.

\section{Purpose}

To reveal how skin $T$ cells age from the viewpoint of density, diversity and function.

\section{Methods}

- Total 91 skin specimens and 79 blood samples were collected from Japanese and Swedish subjects from surgical discards and pre-operation blood tests, respectively.

- T cell density was calculated by flow cytometry analysis (FC, blood) and immunefluorescense analysis (IF, skin). Surface markers of the isolated T cells were analyzed by FC.

- As for anti-pathogen responses, PBMC were co-cultured with heat-killed S.au or C.alb for 7 days and cytokine production of $T$ cells was analyzed by FC. Skin specimens were also injected with the above heat-killed pathogens and T cells were isolated in 14 days for analyzing cytokine production profiles.

- T cell receptor repertoire analysis was conducted by Repertoire Genesis Inc.(Osaka, Japan) on $7 \mathrm{~mL}$ blood and $9 \pi \mathrm{mm}^{2}$ skin specimens from 16 subjects of various ages.

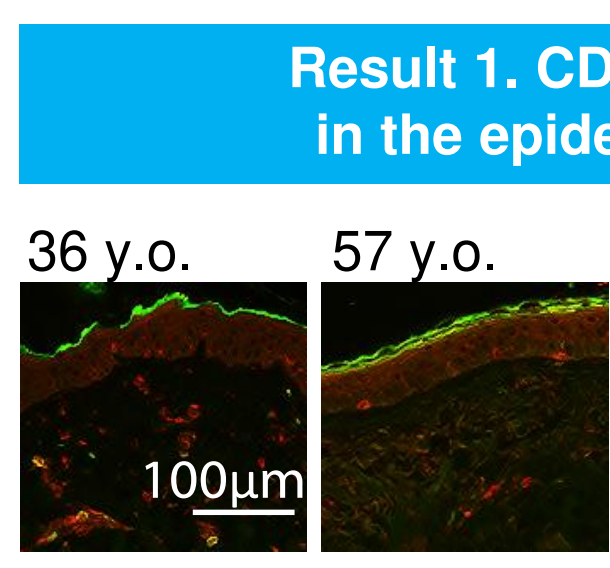

\section{CD49a ${ }^{+}$CD8 TRM are increased \\ ernis of elderly individuals}

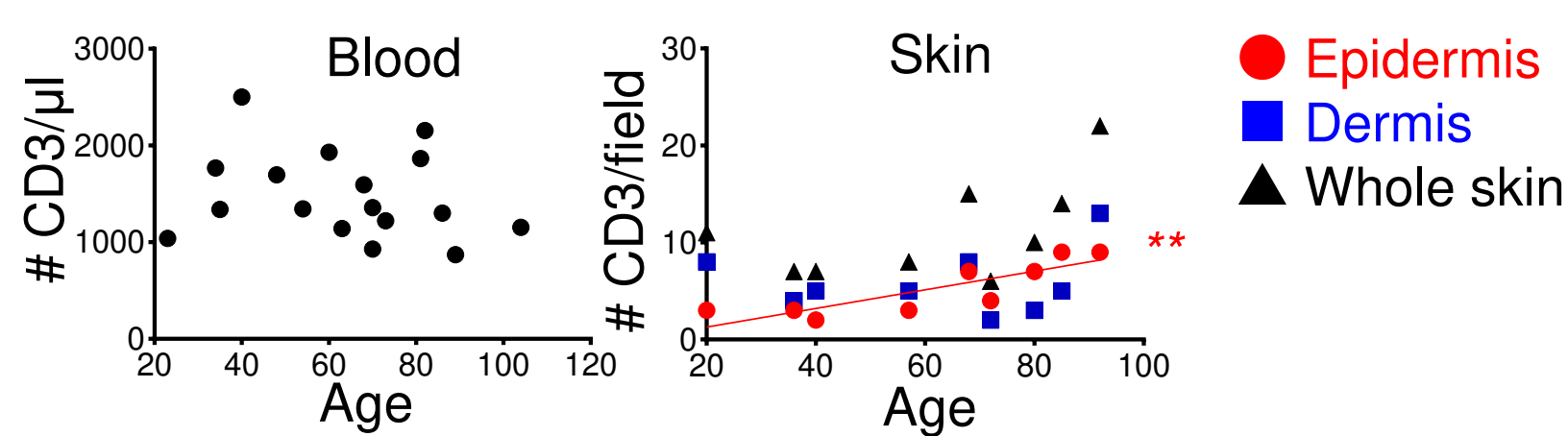

While the density of blood T cells do not change, skin T cells, especially epidermal $\mathrm{T}$ cells, increase through aging (blood: $\mathrm{n}=17$, skin: $\mathrm{n}=9$ from Japanese subjects). ${ }^{* *} p<0.01$.
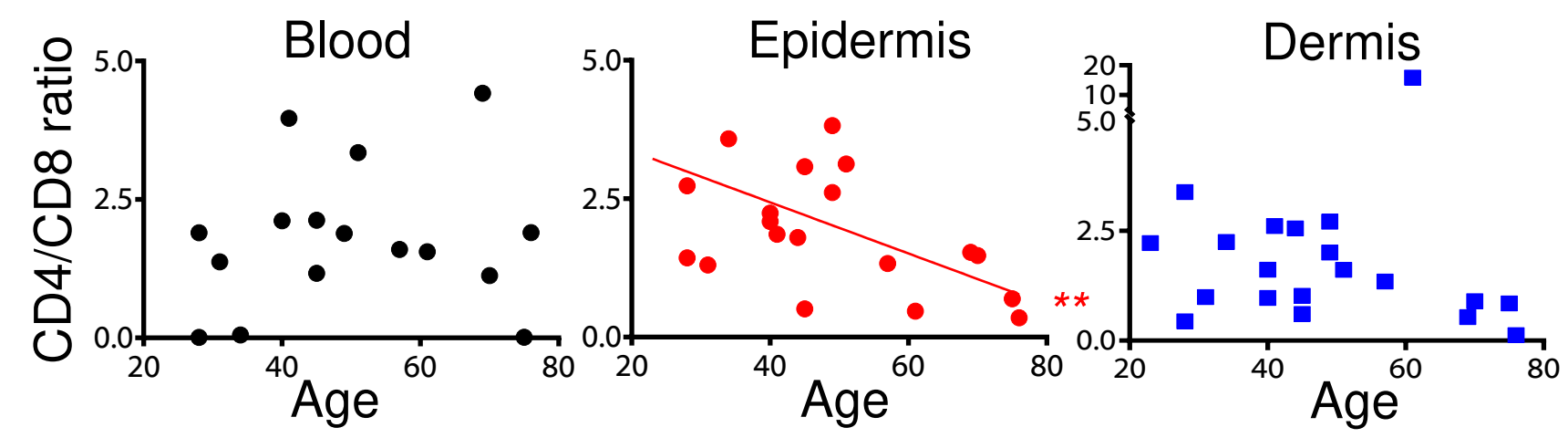

Epidermal T cells in elderly subjects are CD8 dominant (blood: $\mathrm{n}=$ 16, skin: $\mathrm{n}=20$ from Swedish subjects). ${ }^{* *} p<0.01$.
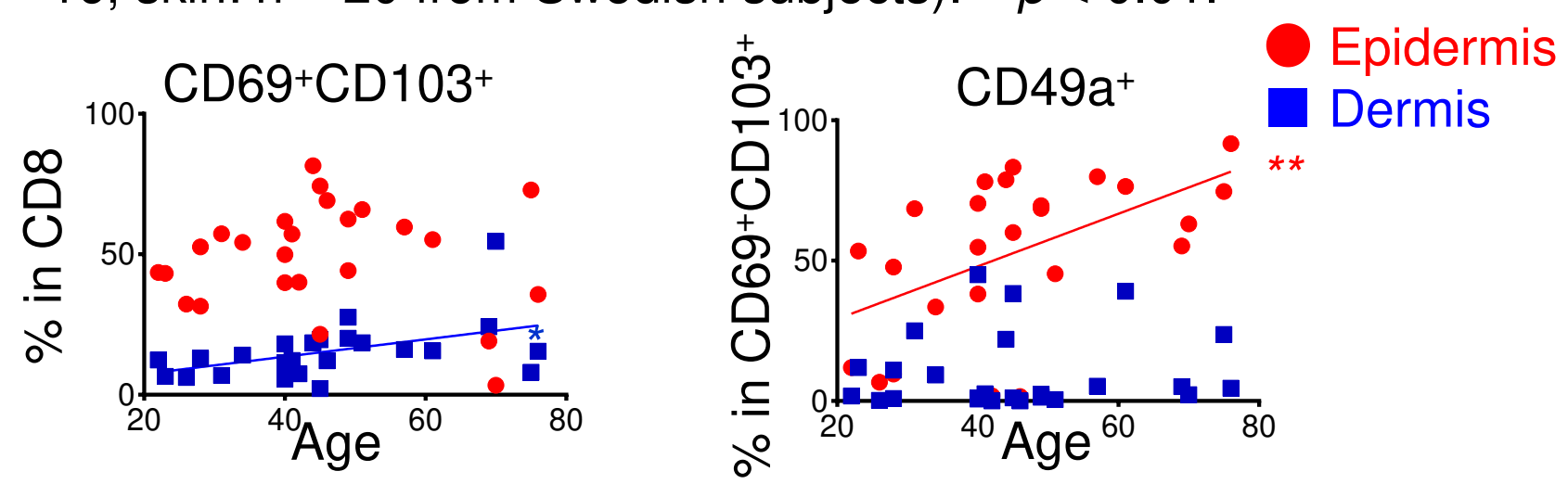

Among CD8 T cells, dermal $\mathrm{CD}^{2}{ }^{+} \mathrm{CD} 103^{+}$and epidermal $\mathrm{CD} 49 \mathrm{a}^{+} \mathrm{CD} 69^{+} \mathrm{CD} 103^{+}$cells are accumulated in elderly subjects

$\left(\mathrm{n}=25\right.$ from Swedish subjects). ${ }^{*} p<0.05,{ }^{* *} p<0.01$.
Result 2. Anti-pathogen responses decline with age in blood but not skin $\mathrm{T}$ cells
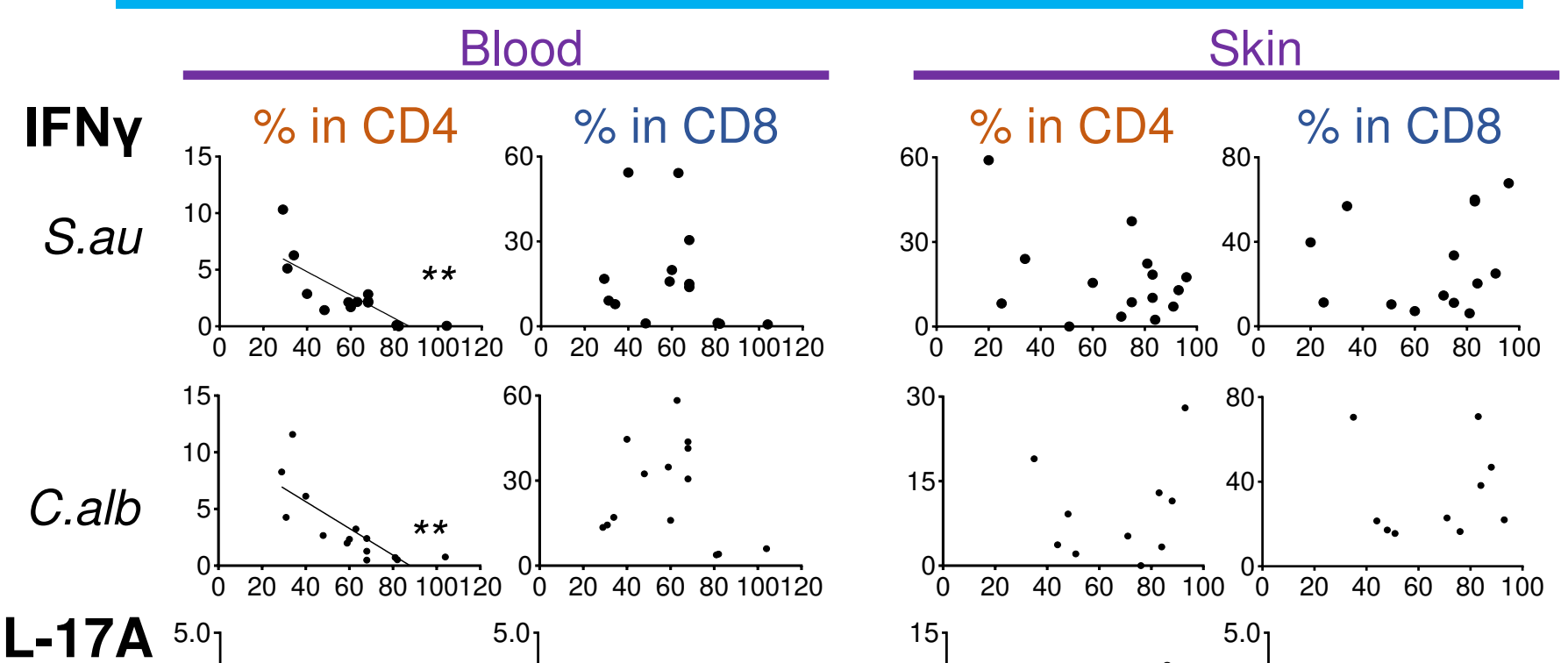

IL-17A

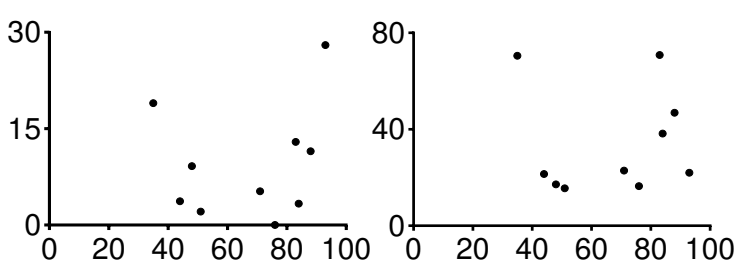

S.au

C.alb
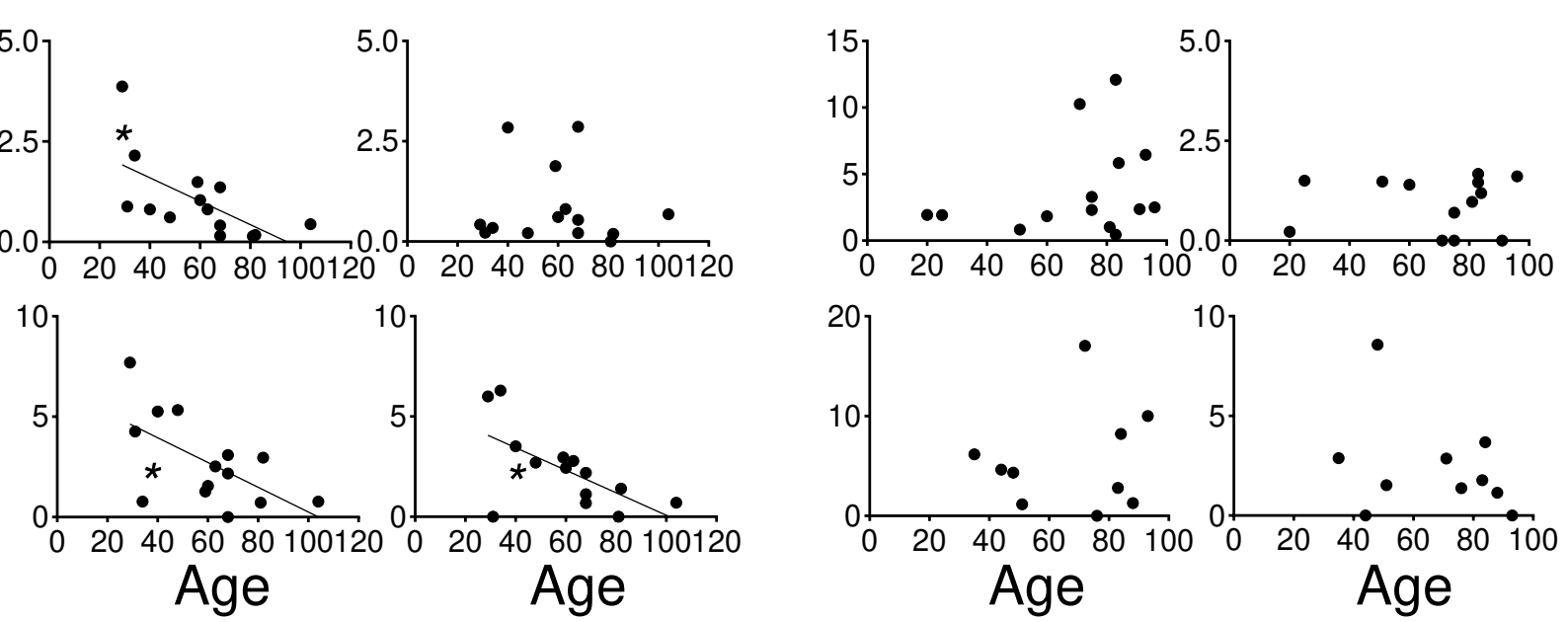

IL-17A and IFNy production from blood T cells are reduced in response with heat-killed S.au and C.alb according to aging. On the other hand, cytokine production from skin $T$ cells are not diminished in elderly subjects reacting with the same pathogens (blood: $n=14$, skin: $n=15$ (S.au) and $\mathrm{n}=10$ (C.alb) from Japanese subjects). ${ }^{*} p<0.05,{ }^{* *} p<0.01$.

Result 3. T cells from skin but not blood maintain diversity in older individuals
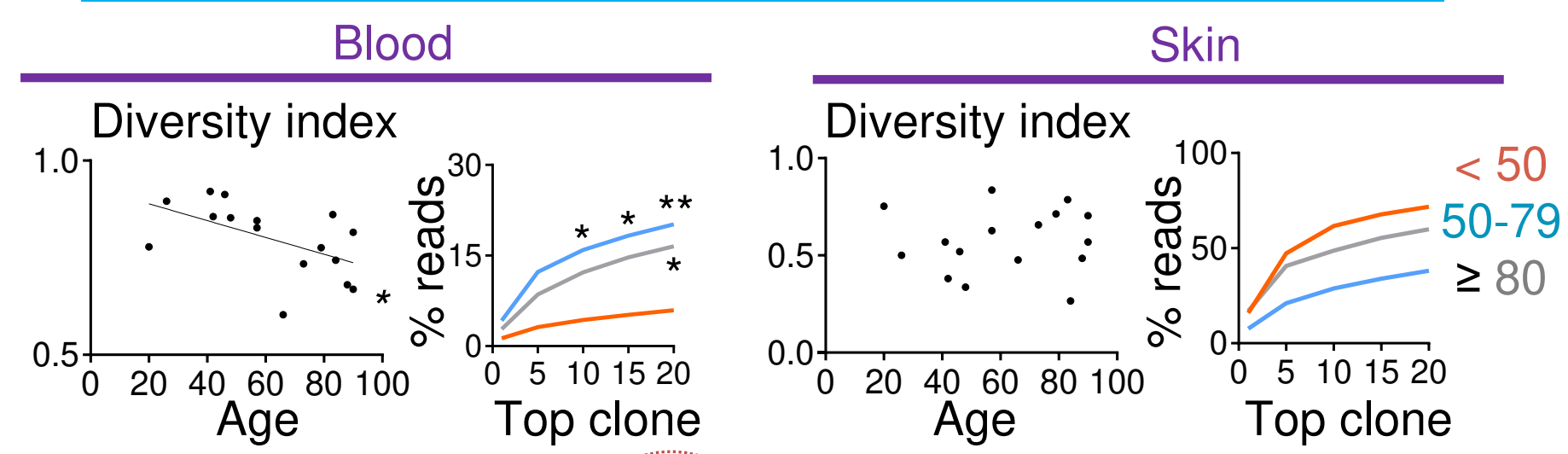

Clones found

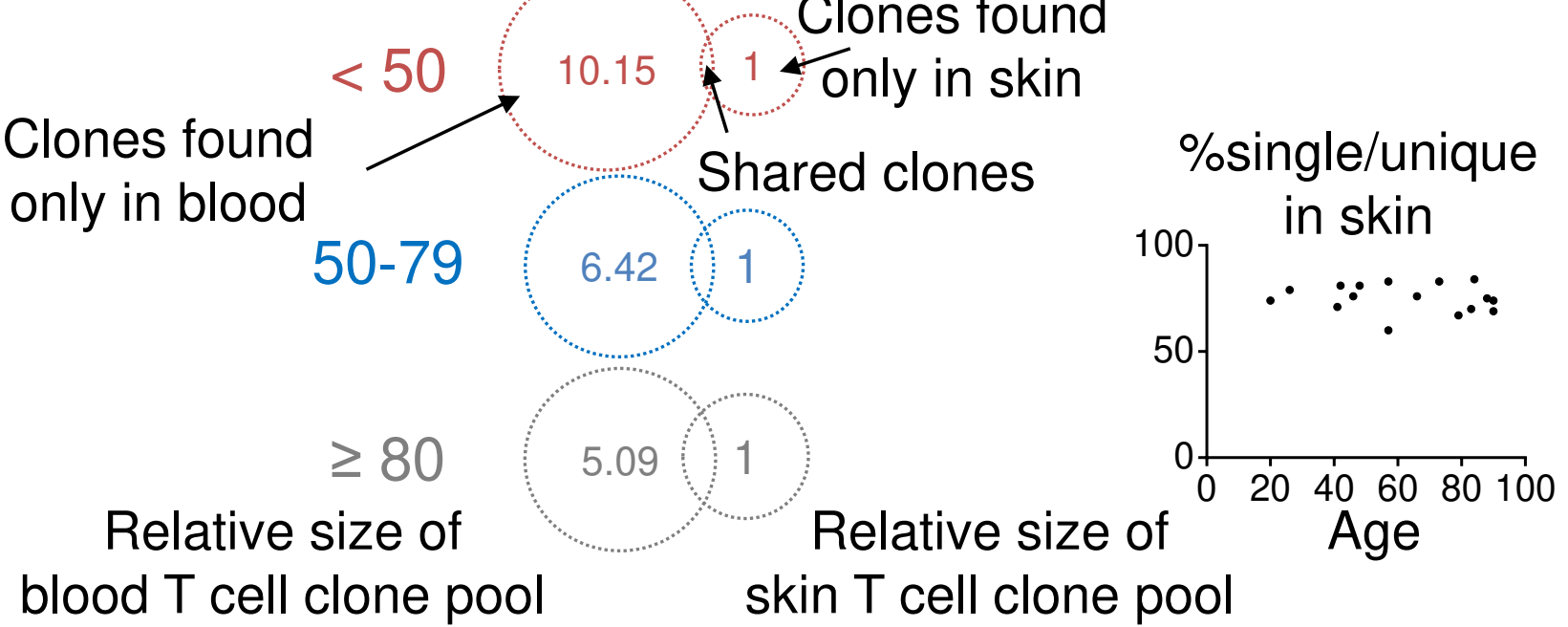

blood T cell clone pool skin T cell clone pool

Top Left: A diversity index (Pielou's evenness) of blood, not skin, decreases by aging. $\mathrm{N}=16$ from Japanese subjects. Top Right: The mean occupancy of top 1 20 most frequently detected TCR clones, which are expanding clones, is high in elderly blood and low in elderly skin. The same 16 subjects are divided into three age groups $(<50$ y.o. (orange): $n=6,50-79$ y.o. (blue): $n=5, \geq 80$ y.o. (gray): $n=5$ ). ${ }^{*} p<0.05$, ${ }^{* *} p<0.01$. Bottom left: The percentage of clones whose copy number is single in total unique clones is over $60 \%$ in skin of all ages. These data suggest that skin $\mathrm{T}$ cells are kept diverse during aging while blood $\mathrm{T}$ cells show oligoclonal expansion. The illustration suggests the estimated ratio of the size of TCR clone pool in blood and skin calculated by the average number of shared clones between blood and skin and the occupancy of the shared clones per blood or skin unique clones. The ratios of blood T cell pool and skin $T$ cell pool are 10.15:1 in $<50$ y.o. and 5.09:1 in $\geq 80$ y.o., indicating that skin T cell diversity is about twice better maintained.

\section{Conclusions}

